\title{
Bifurcations of flame filaments in chaotically mixed combustion reactions
}

\author{
Shakti N. Menon and Georg A. Gottwald* \\ School of Mathematics 83 Statistics, University of Sydney, \\ New South Wales 2006, Australia
}

\begin{abstract}
We study the behaviour of steady-state solutions of a two-component flame filament system subject to chaotic mixing. This system exhibits a saddle-node bifurcation at a critical Damköhler number. We analyze the system through a one-dimensional phenomenological lamellar model. We present a nonperturbative technique, which allows us to describe the behaviour of the reduced lamellar model near the saddle node bifurcation. The influence of the Lewis number on the solution behaviour is investigated. We present a simple empirical formula for the wave speed valid for large Damköhler and large Lewis numbers. This formula allows us to describe the solution far away from the bifurcation. Numerical simulations show good agreement with the results.
\end{abstract}

PACS numbers: 47.54.-r, 82.40.Ck, 47.51.+a, 82.33.Vx, 05.45.-a

\footnotetext{
*corresponding author: gottwald@maths.usyd.edu.au
} 


\section{Introduction}

The field of active chaotic flows is a rapidly developing interdisciplinary area of nonlinear science, which focuses on the effect of chaotic advection on chemical and biological reactions [1]. It has been observed that imperfect mixing in diffusive nonlinear systems (such as autocatalytic systems) can give rise to new and unexpected phenomena [2]. A recent overview is given in [3]. The chaotic dynamics induced by advecting time-dependant flows manifests itself in the distribution of fluid parcels along fractal patterns leading to the formation of filamental structures. In the case of active processes, reactive tracers are advected with these filaments, and the increase in their surface area has an effect on the kinetics of the reaction. This often has important consequences in wide ranging fields. Besides in the case of combustion, where mixing-induced bifurcations may lead to unwanted termination of the reaction, the study of chaotically stirred reaction-diffusion systems also has important consequences for a number of environmental processes, such as depletion of ozone by chlorine filaments [4] and development of plankton patchiness [5, 6, 7, 8, 9, 10]. It has been observed that fluid mixing has a significant effect on combustion processes and in particular on flame filamental structures $[11,13,14,15,16,17,18]$. The quenching of a flame is strongly dependant on the Damköhler number $D a$, which is the ratio between the advective and chemical time-scales. Much insight has been gained by reducing the typically 2-dimensional partial differential equations describing combustion to so called lamellar models. The idea behind this reduction is as follows. A chaotic stirring flow is characterized by hyperbolic regions of stretching and compression, which lead to filamentation of the reactant. The stretching is directed along the filament whereas the compression is directed transversal to the filament. Along the filament the concentration of the reactant is approximately homogeneous. Hence, in order to study the dynamics of such a filament, one may consider only the dynamics of the cross-section of a filament which is aligned with the direction of compression. The dimensionality of the problem is therefore reduced to one spatial dimension. In lamellar models, steady-state solutions are formed as the balance of the inwards-directed compression and the outwards-directed diffusive propagation of a reactant. If the compression is too large, the reaction will be suppressed. Lamellar models have been widely used in different physical contexts $[6,8,18,19,20,21,22,23,24]$. A critical account on such reductions is given in [25]. For a different approach to this problem see [26].

We study in this work the influence of chaotic stirring on combustion waves. One particularity for problems involving combustion waves is the strong dependence of the reaction rate on the temperature. This is usually modelled by an Arrhenius term [30]. A combustion wave can be (at least for low stirring rates) divided into three distinct regions. Ahead of the combustion waves, in the so called preheat zone, the temperature is low and the reactant has not been burnt. When the temperature increases and becomes sufficiently high, the reaction rate increases exponentially and and the reactant is quickly burnt under heat release. This takes place in a narrow steep region called the reaction zone. Behind the front there is the product zone where all reactant is burnt, no reaction occurs and the temperature is constant. The behaviour of combustion waves is also 
characterized by the Lewis number. The Lewis number Le is a non-dimensional number which measures the ratio of the diffusivity of the temperature with the diffusivity of the reactant. Systems with a large Lewis number are often called "solid" whereas systems with low Lewis numbers are called "gaseous".

The stirred system is further characterized by the dimensionless Damköhler number $D a$. The Damköhler number Da measures the ratio of the time scale of the stirring flow with the time scale of the reaction. The smaller $D a$ is the faster the stirring is compared to the reaction. Chaotic stirring may lead to an unwanted termination of the reaction. This combustion wave propagation failure occurs as a saddle node bifurcation. A saddle node bifurcation - generic for chaotically stirred reaction-diffusion systems - was observed in $[18,19]$ for a combustion system at a critical Damköhler number $D a_{c}$. The bifurcation point corresponds to the "quenching point", below which the stirring rate exceeds the rate at which the fuel burns and the flame is extinguished. The critical value of the Damköhler number $D a_{c}$ depends on the Lewis number. It is this bifurcation that we are mainly concerned with in this work. In [18] an asymptotic analysis was developed for various limiting cases, where the ordinary differential equations describing the stationary fronts were simplified. A numerical integration of these asymptotic equations was able to determine the critical Damköhler number fairly well. However, the pulse behaviour near the saddle node has not yet been described accurately. To fill this gap we will employ a nonperturbative, non-asymptotic technique that was developed for excitable media [27] and which had successfully been employed for chaotically stirred autocatalytic and bistable reaction diffusion dynamics [28, 29].

In the next section, we present the lamellar model used to describe the flame filament system. In Section 3, the nonperturbative, non-asymptotic technique is reviewed. In Section 4, we use this method to describe the behaviour near the saddle node for different values of $L e$, and in Section 5 we study the case of slow stirring (for solutions far away from the bifurcation point). We conclude with a summary in Section 6 .

\section{The flame filament model}

We study a simple combustion system in which the conversion of a fuel $C$ of concentration $c$ and absolute temperature $T$, to an inert product $P$, is modelled as a first order process through the reaction $C \rightarrow P$. The model proposed in $[18,19]$ describes the resulting filament structures of such a system when it is subjected to a chaotic stirring flow. The temperature dependence of the reaction rate $k(T)$ is given by an Arrhenius law

$$
k(T)= \begin{cases}k_{0} \exp \left(-\frac{E}{R T}\right) & \text { if } T>T_{i}, \\ 0 & \text { if } T \leq T_{i}\end{cases}
$$

with an ignition temperature $T_{i}$, and an activation energy $E$. Here $R$ denotes the universal gas constant. The cutoff temperature $T_{i}$ allows for a simple solution to the so called cold boundary problem. If $T_{i}=0$, large preheat zones may arise which imply a non-zero 
temperature at the boundary, away from the reaction zone. Introducing this non-zero cutoff allows for a finite box with a cold boundary of $T=0$. This is a purely practical issue introduced to simplify numerical simulations. We note that there is no change to the results we present here if a purely Arrhenius reaction term is used.

The model is then given by

$$
\begin{aligned}
\rho C_{p}\left(\frac{\partial T}{\partial t}-\lambda x \frac{\partial T}{\partial x}\right) & =\kappa \frac{\partial^{2} T}{\partial x^{2}}+q c k(T), \\
\frac{\partial c}{\partial t}-\lambda x \frac{\partial c}{\partial x} & =D \frac{\partial^{2} c}{\partial x^{2}}-c k(T),
\end{aligned}
$$

in the region $-\infty<x<\infty$, where $\rho$ represents the density of the reactant. $C_{p}$ the specific heat capacity, $\kappa$ the thermal conductivity, $D$ the diffusion coefficient, and $k(T)$ is the reaction rate given in (1). The parameter $\lambda$ describes the compression of the filament and can be thought of as the Lagrangian mean strain of the chaotic flow in the contracting direction, and may be given by the absolute value of the negative Lyapunov exponent of this flow. The boundary condition applied to this system is $T \rightarrow T_{a}, c \rightarrow c_{0}$ as $|x| \rightarrow \infty$, where $T_{a}$ is the ambient temperature (assumed to be less than the ignition temperature $T_{i}$ ). We rescale the equations (2) and (3) by introducing nondimensional variables $T^{\prime}=\left(T-T_{a}\right) /\left(T_{b}-T_{a}\right), c^{\prime}=c / c_{0}, t^{\prime}=\lambda t$ and $x^{\prime}=x \sqrt{\lambda / D}$, where $T_{b}$ is the burnt temperature $T_{b}=T_{a}+\left(q c_{0} / \rho C_{p}\right)$. Omitting the primes, we obtain

$$
\begin{aligned}
\frac{\partial T}{\partial t}-x \frac{\partial T}{\partial x} & =L e \frac{\partial^{2} T}{\partial x^{2}}+\operatorname{DacK}(T), \\
\frac{\partial c}{\partial t}-x \frac{\partial c}{\partial x} & =\frac{\partial^{2} c}{\partial x^{2}}-\operatorname{DacK}(T) .
\end{aligned}
$$

Here, we have introduced the Damköhler number $D a=k_{0} / \lambda$, and the Lewis number $L e=\kappa /\left(\rho C_{p} D\right)$. The Damköhler number measures the ratio of the timescale of the stirring flow $1 / \lambda$ with the time scale of the reaction $1 / k_{0}$. For $L e \neq 1$ the diffusivities of temperature and concentration are different. The rescaled system now has the boundary conditions $T \rightarrow 0, c \rightarrow 1$ as $|x| \rightarrow \infty$, while the reaction rate is now given by

$$
K(T)= \begin{cases}\exp \left(-\frac{1}{\epsilon((1-\beta) T+\beta)}\right) & \text { if } \quad T>\bar{T}_{i}, \\ 0 & \text { if } T \leq \bar{T}_{i}\end{cases}
$$

with parameters

$$
\epsilon=\frac{R T_{b}}{E}, \quad \beta=\frac{T_{a}}{T_{b}}, \quad \bar{T}_{i}=\frac{T_{i}-T_{a}}{T_{b}-T_{a}} .
$$

We are particularly interested in steady-state solutions of (4) and (5) which are given as solutions of the ordinary differential equations

$$
\begin{aligned}
L e \frac{d^{2} T}{d x^{2}}+x \frac{d T}{d x}+\operatorname{Dac} K(T) & =0 \\
\frac{d^{2} c}{d x^{2}}+x \frac{d c}{d x}-\operatorname{Dac} K(T) & =0 .
\end{aligned}
$$


We recall that a stationary front is given through a balance of the front velocity $V$ with the velocity of the chaotic stirring $x$. The front is a steady-state with a zero velocity when $V=x$. For all following numerical simulations we use $\beta=0.1, \epsilon=1$ and $\bar{T}_{i}=0.001$, unless explicitly stated otherwise.

It has been observed that the temperature and concentration profiles of these steadystates depend strongly on $L e$. In the limiting cases of small and large Lewis numbers the solution could be studied through an asymptotic analysis [18]. We will focus here on applying a nonperturbative method, which does not require asymptotic expansions, to describe the solution behaviour close to the bifurcation and far away from it. Instead of obtaining a set of reduced ordinary differential equations which subsequently had to be integrated numerically, we will obtain a set of algebraic equations determining the parameters of the solutions and the actual bifurcation point. In the next Section we will outline this nonperturbative method.

\section{The Nonperturbative method}

In the study of critical wave propagation in reaction-diffusion systems, it is observed that asymptotic techniques break down near the bifurcation point. This is due to the fact that the pulse shape in this region is approximately bell-shaped (see Fig. 1,5 and 7). Close to the bifurcation the solution can not be separated into inner and outer regions, which have been employed by asymptotic theories. Hence, a new method was developed in order to determine the bifurcation point and the shape of near-critical pulses for critical wave propagation in excitable media [27]. This method has since been successfully applied to chaotically stirred autocatalytic and bistable systems [28, 29].

The idea is to restrict the solutions of the system under consideration, i.e. (4)-(5) or (6)-(7), to a subspace of specified test functions $C\left(\eta, a_{i}\right)$, which are parameterised by $a_{i}$. For example we can use a Gaussian as a test-function, in which case the parameters are given by its amplitude and its inverse pulse width. These parameters are then determined by minimizing the error made by the restriction to the subspace defined by the test function ansatz. This is achieved by projecting the full partial differential equations onto the tangent space of the restricted subspace, which is spanned by the partial derivatives of the test functions with respect to the parameters $a_{i}$. We set the integral of the products of Eq. (4) and (5), or (6) and (7), with the basis functions of the tangent space to zero. This will lead to algebraic equations for the parameters $a_{i}$, and also yield the critical Damköhler number $D a_{c}$.

The question is which functions to use as test functions. Numerical solution of the system (4)-(5) reveal that for lower values of $D a$ the profiles of $T$ (and $1-c$ ) are pulse-like, and are well approximated by bell-shaped functions, such as Gaussians (see Fig. 1,5 and 7). For higher values of $D a$ the solutions are plateau-like with a clear separation of two flat regions by a steep front, and can be approximated by test functions that show this 
behaviour, such as tanh-functions (see Fig. 1,5 and 7). However, it is to be noted that the exact behaviour of the solution at any given value of $D a$ is strongly dependent on the value of $L e$, and in the following Sections, we shall examine some of these trends. The general form of our solution is

$$
T(x)=\Theta\left(x, a_{i}\right) \quad \text { and } \quad c(x)=1-\Omega\left(x, a_{i}\right) .
$$

We may choose the test functions $\Theta\left(x, a_{i}\right)$ and $\Omega\left(x, a_{i}\right)$ to be either bell-shaped or plateaulike depending on the nature of the solution at the corresponding values of $D a$ and $L e$.

Close to the bifurcation point the solutions are bell-shaped. The choice for the actual functional form for these bell-shaped functions is arbitrary. There is no asymptotic argument that can be made to favour one functional form over the others. Unless stated otherwise, we shall choose Gaussians

$$
\begin{array}{lll}
\Theta\left(x, a_{i}\right)=f_{0} \exp \left(-\eta^{2}\right) & \text { with } & \eta=w x, \\
\Omega\left(x, a_{i}\right)=g_{0} \exp \left(-\zeta^{2}\right) & \text { with } & \zeta=v x .
\end{array}
$$

Here $a_{i}=\left\{f_{0}, w, g_{0}, v\right\}$. But one may equally use $\operatorname{sech}^{n}$-functions or other such bellshaped functions for $\Theta$ and $\Omega$. We will see later that the results do not significantly depend on the actual functional form of the bell-shaped function - indicating the absence of an asymptotic theory which may be able to deduce the functional form.

For plateau-like solutions at large Damköhler numbers we use

$$
\begin{aligned}
& \Theta\left(x, a_{i}\right)=\frac{f_{0}}{2}\{\tanh (\eta+\nu w)-\tanh (\eta-\nu w)\} \\
& \Omega\left(x, a_{i}\right)=\frac{g_{0}}{2}\{\tanh (\zeta+\mu v)-\tanh (\zeta-\mu v)\},
\end{aligned}
$$

where again $\eta=w x$ and $\zeta=v x$, and now $a_{i}=\left\{f_{0}, w, \nu, g_{0}, v, \mu\right\}$. Here $\nu$ and $\mu$ are the widths of the corresponding test functions, and $w$ and $v$ measure the steepness of the reaction zone. By choosing (10) we ignore the problem of a preheat zone which would requires us to take two composite tanh-functions as test functions, each equipped with two different widths $w_{1,2}$ and $v_{1,2}$. This was observed numerically for large Damköhler numbers and also for small Lewis numbers, when the reaction zone becomes narrow. In passing we note that for such cases the reaction term $c K(T)$ in (6) and (7) is zero except in the narrow reaction zone. Outside this small steep region either $c$ or $T$ are close to zero. The stationary equations can therefore be approximated by the uncoupled equations $0=L e T_{x x}+x T_{x}$ and $0=c_{x x}+x c_{x}$, which are solved by error functions.

As described above, we restrict the solutions to the subspace of the test functions $\Theta(\eta)$ and $\Omega(\zeta)$, and determine the free parameters by minimizing the error made by this restriction. We do this by projecting equation (4) onto the tangent space of the restricted subspace spanned by $\partial T / \partial a_{i}$, and similarly, (5) onto the space with basis functions $\partial c / \partial a_{i}$, where $a_{i}$ are the parameters of the tangent space. If we choose a combination of bellshaped test functions (9), the free parameters are $a_{i}=\left\{f_{0}, w, g_{0}, v\right\}$, and the tangent 
space of the space of test functions is spanned by the basis functions

$$
\frac{\partial T}{\partial f_{0}}=\frac{\Theta}{f_{0}}, \frac{\partial T}{\partial w}=\frac{\eta \Theta_{\eta}}{w}, \frac{\partial c}{\partial g_{0}}=-\frac{\Omega}{g_{0}}, \frac{\partial c}{\partial v}=-\frac{\zeta \Omega_{\zeta}}{v} .
$$

If we choose a plateau-like solution (10), we have the additional free parameters $\nu$ and $\mu$, and the basis functions of the tangent space are

$$
\begin{gathered}
\frac{\partial T}{\partial f_{0}}=\frac{\Theta}{f_{0}}, \frac{\partial T}{\partial w}=\frac{1}{2}\left(\frac{\eta}{w}\left(s_{1}-s_{2}\right)+\nu\left(s_{1}+s_{2}\right)\right), \quad \frac{\partial T}{\partial \nu}=\frac{w}{2}\left(s_{1}+s_{2}\right), \\
\frac{\partial c}{\partial g_{0}}=-\frac{\Omega}{g_{0}}, \frac{\partial c}{\partial v}=-\frac{1}{2}\left(\frac{\zeta}{v}\left(s_{3}-s_{4}\right)+\mu\left(s_{3}+s_{4}\right)\right), \frac{\partial c}{\partial \mu}=-\frac{v}{2}\left(s_{3}+s_{4}\right),
\end{gathered}
$$

where $s_{1}=\operatorname{sech}^{2}(\eta+\nu w), s_{2}=\operatorname{sech}^{2}(\eta-\nu w), s_{3}=\operatorname{sech}^{2}(\zeta+\mu v)$ and $s_{4}=\operatorname{sech}^{2}(\zeta-\mu v)$. Hence, depending on our choices for the test functions, the basis functions of the tangent space are represented by (11) or by (12). The projection is done by integrating the product of the equations (6) and (7) with the corresponding basis functions over the $\eta$ and $\zeta$ domains. Minimizing the error caused by restricting the solutions requires this projection to be zero. Inserting (8) into (6) and (7), we obtain

$$
\begin{aligned}
w^{2} L e \Theta_{\eta \eta}+\eta \Theta_{\eta}+D a(1-\Omega) K(\Theta) & =0 \\
v^{2} \Omega_{\zeta \zeta}+\zeta \Omega_{\zeta}+D a(1-\Omega) K(\Theta) & =0 .
\end{aligned}
$$

The projections of equations (13) and (14) onto the tangent space with basis functions given in (11) or (12), depending on our choice of test functions, lead to the following conditions

$$
\left\langle w^{2} L e \Theta_{\eta \eta}+\eta \Theta_{\eta}+D a(1-\Omega) K(\Theta) \mid \partial T / \partial a_{i}\right\rangle_{\eta}=0,
$$

where the brackets indicate integration over the whole $\eta$ domain, and

$$
\left\langle v^{2} \Omega_{\zeta \zeta}+\zeta \Omega_{\zeta}+D a(1-\Omega) K(\Theta) \mid \partial c / \partial b_{i}\right\rangle_{\zeta}=0
$$

where the brackets indicate integration over the whole $\zeta$ domain. These are four (in the case of bell-shaped functions) or six (in the case of plateau-like functions) equations for the respective four or six parameters. However, for any of the choices of basis functions, the resulting equations (15)-(16) are transcendental equations for the parameters, due to the $K(\Theta)$ term. We therefore integrate this system numerically and use root finding methods to obtain the free parameters.

In the following Section, we shall use this nonperturbative method to study the solution near the bifurcation point for different values of the Lewis number. We will then study the solution far from $D a_{c}$ in Section 5 .

\section{The solution near the saddle node}

Numerical simulations of the system (6)-(7) reveals the existence of a saddle node bifurcation in which a stable solution branch collides with an unstable branch when the 
Damköhler number $D a$ is decreased to a critical value $D a_{c}$. As mentioned in Sec. 3 , close to the bifurcation point we may approximate the solutions of $T$ and $c$ by bell-shaped functions. We therefore use such test functions in our ansatz (8) to solve (15)-(16). We now show a comparison of the nonperturbative test function results with the corresponding results from the numerical solution of the full system (6)-(7), obtained via shooting methods for corresponding $D a$ and $L e$.

\subsection{Small Lewis numbers}

For small Lewis numbers the reaction zone is narrow, and within the product zone temperatures which are higher than the burnt temperature $T_{b}$ (i.e. higher than 1 in our rescaled model) can be obtained without much consumption of the reactant. However, with increasing Damköhler number the temperature in the product zone will approach the burnt temperature. In Fig. 1, we show the profiles of $T$ and $c$ for $L e=0.1$, obtained from the numerical solution of (6)-(7). We observe that the solutions of both $T$ and $c$ are pulse-like near the bifurcation point, but as $D a$ increases we see that the stable solutions for both slowly become plateau-like. As we move away from $D a_{c}$, the stable solution for $T$ first increases in amplitude, and then slowly decreases upon varying $D a$. On the other hand, the unstable solutions for both $T$ and $c$ remain pulse-like (with decreasing steepness) as we move further and further away from the saddle node.

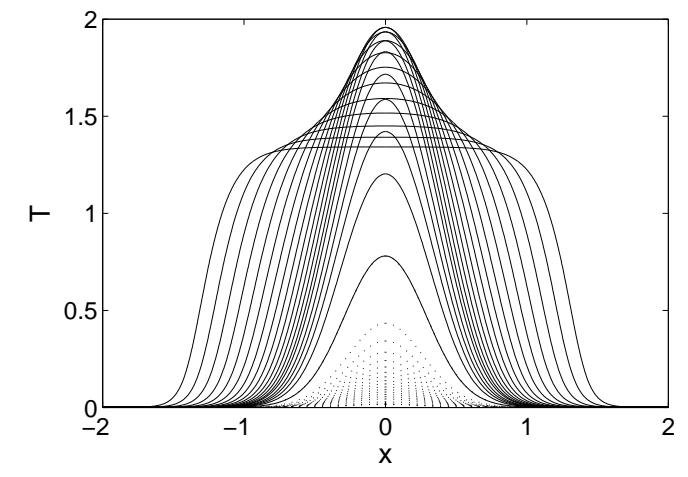

$(a)$

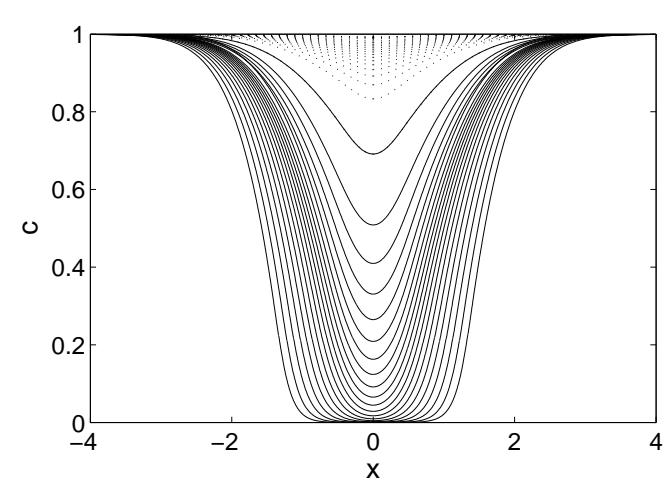

$(b)$

Figure 1: The steady solutions of the flame filament system (6)-(7) for $L e=0.1$. Both the stable solutions (denoted by solid lines) and the unstable solutions (denoted by dotted lines) are shown for logarithmically spaced values of $D a$ between $D a=4.62\left(\approx D a_{c}\right)$ and $D a=150$. (a) Profiles of $T$. (b) Profiles of $c$.

To model the pulse-like behaviour near the bifurcation point, we use bell-shaped test functions (9) as our basis functions. As stated earlier, the exact functional form of our bellshaped function is arbitrary, and to illustrate this, we shall choose Gaussians (9) as well as sech ${ }^{2}$-functions with $\Theta(\eta)=f_{0} \operatorname{sech}^{2}(\eta)$ and $\Omega(\zeta)=g_{0} \operatorname{sech}^{2}(\zeta)$ as our test functions. From the numerical solution of the equations (15)-(16) with these basis functions, we can obtain values for the amplitudes and inverse widths for our test functions over a range of 
$D a$. We compare these results with the amplitudes of $T$ and $c$ obtained from numerical simulations of the full system (6)-(7). The full system of ordinary differential equations is solved by a standard shooting method where we shoot from $x=0$ to $x=\infty$.

As seen in Fig. 2, near the saddle node we find very good agreement between the amplitudes $f_{0}$ and $1-g_{0}$ obtained with these bell-shaped test functions, and the amplitudes obtained by simulating the full ordinary differential equations (6) and (7). We observe that there is not much difference between the set of results obtained using Gaussians, and those obtained using $\operatorname{sech}^{2}$-functions. As we move away from the saddle node the fits start to get worse along the stable branch, which is to be expected as the solutions of $T$ and $c$ move away from pulse-like profiles as the Damköhler number increases. Also, as expected, we find excellent agreement along the unstable branch even for larger $D a$, as both the solutions of $T$ and $c$ remain pulse-like. This is true for all Lewis numbers (cf. Fig. 6 and Fig. 8). In Fig. 3 we show the behaviour of the inverse pulse widths $w$ and $v$ of the temperature and the concentration respectively. The inverse pulse width exhibits the same functional dependence with $D a$ typical for a saddle-node, at the same value of $D a_{c}$, as the amplitude in Fig. 2. Again good agreement between the test function approach and the actual results of a simulation of the full system is seen. The pulse width for the actual solutions of the full system (6) and (7) were determined by measuring the extent $\bar{x}$ of the pulse measured from $x=0$ at which its value has dropped to half its value. For a Gaussian, this implies that $w=\sqrt{\ln (2)} / \bar{x}$.

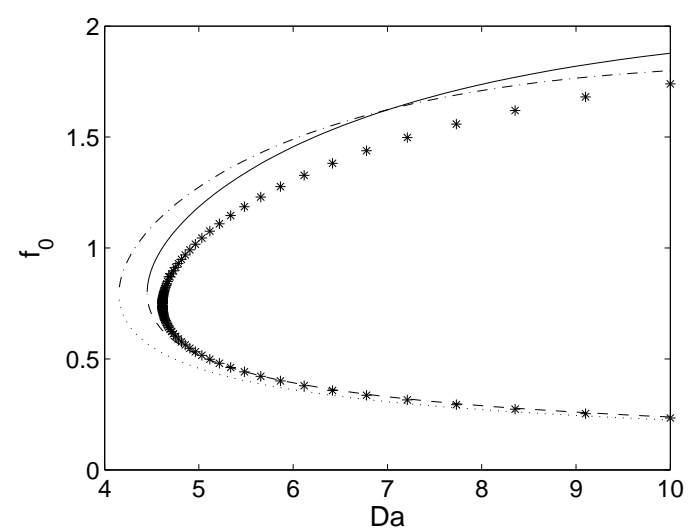

(a)

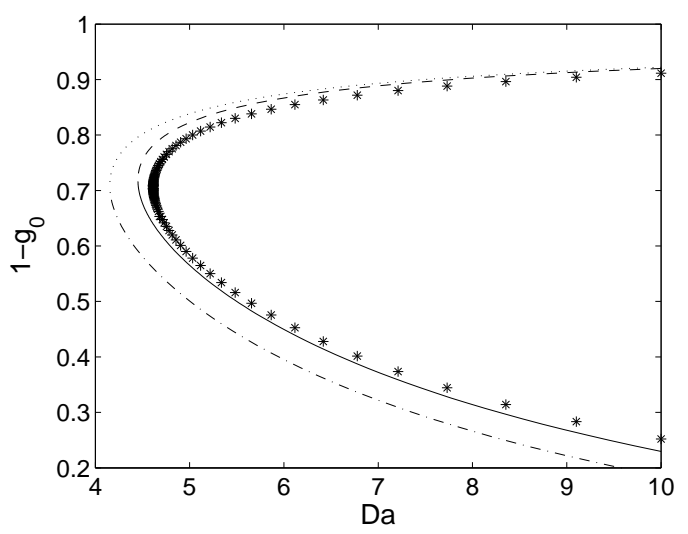

(b)

Figure 2: Comparison of the numerical results for the amplitude, obtained using the nonperturbative method with bell-shaped test functions (9), with the results for the full system of ordinary differential equations (6) and (7) obtained via shooting for $L e=0.1$ (denoted by stars). We use Gaussians (denoted by a solid line and a dashed line for the stable and unstable branches respectively), as well as sech ${ }^{2}$-functions (denoted by a dashed-dotted line and a dotted line for the stable and unstable branches respectively) as our test functions. (a) The amplitude for the temperature, $f_{0}$, versus $D a$. (b) The amplitude for the concentration, $1-g_{0}$, versus $D a$. 


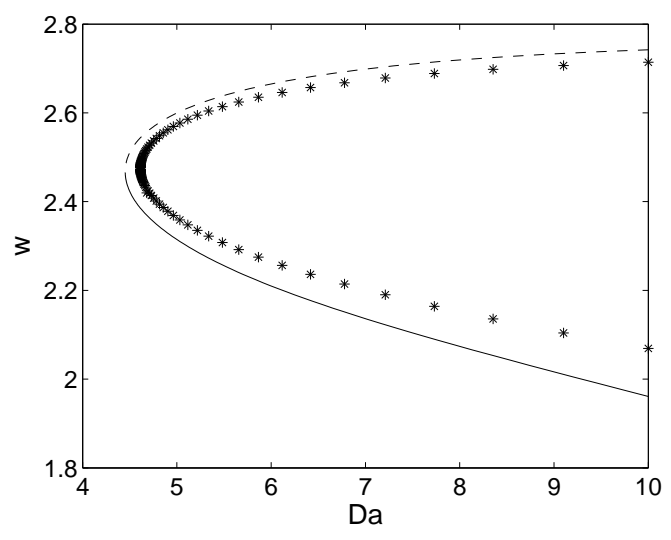

$(a)$

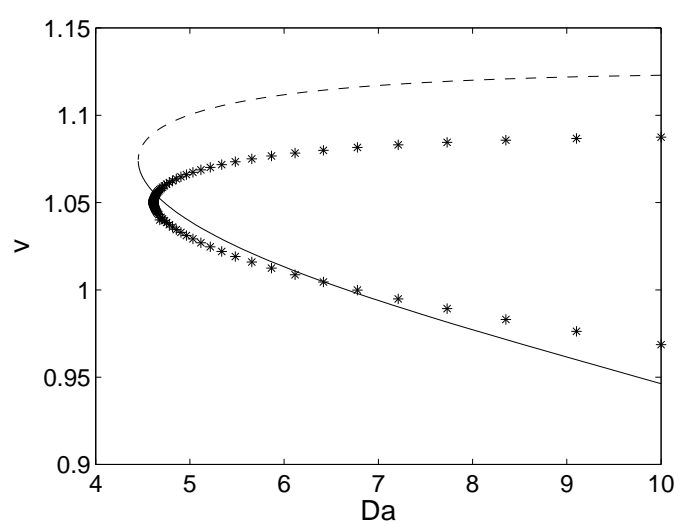

$(b)$

Figure 3: Comparison of the numerical results for the inverse pulses widths, obtained using the nonperturbative method with bell-shaped test functions (9), with the results for the full system of ordinary differential equations (6) and (7) obtained via shooting for $L e=0.1$ (denoted by stars). We use Gaussians (denoted by a solid line and a dashed line for the stable and unstable branches respectively) as our test functions. (a) The inverse pulse width for the temperature, $w$, versus $D a$. (b) The inverse pulse width for the concentration, $v$, versus Da. Note the different scales in (a) and (b).

We note that the combination of tanh-functions (10) which were initially designed to approximate plateau-like solutions can also be used to approximate pulse-like solutions for sufficiently small $\nu$ (or $\mu$ respectively). However, since the ansatz (10) involves 6 parameters (as opposed to 4 parameters for the bell-shaped functions (9)), and as root-finding becomes more and more sensitive to a correct initial guess in higher dimensions, we use bell-shaped functions (11) with less computational effort. However, the tanh-functions are very well suited to reproduce the observed increase of the temperature within the product zone with increasing Damköhler number and the subsequent decrease. This is depicted in Fig. 4. Note that for this range of Damköhler numbers the combustion wave has not reached a plateau-like shape (see Fig. 1). 


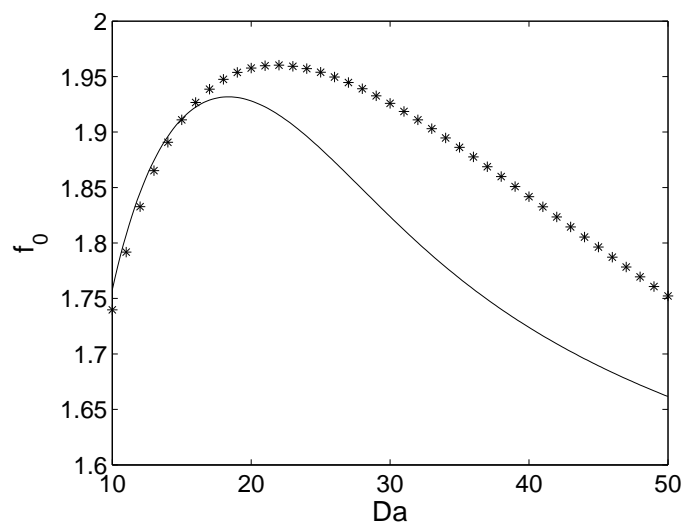

Figure 4: Comparison of the numerical results describing the behaviour of the stable branch of $T$ in the range $10<D a<50$ for $L e=0.1$. The results for the amplitude, $f_{0}$, obtained using the nonperturbative method with basis functions (10) are denoted by a solid line, and the results obtained via shooting of the full system of ordinary differential equations (6) and (7) are denoted by stars.

\subsection{Equal diffusion: $L e=1$}

The case $L e=1$ has attracted much attention because it allows for a great simplification due to the relation $T=1-c$. The two equations (6)-(7) collapse into one single equation. We therefore only need one test function to describe the full behaviour of the system. For this case, the profiles of $T$ and $c$ obtained from numerical simulations of (6)-(7) are shown in Fig. 5. Again we see that along the stable branch both the solutions are pulse-like near the bifurcation point, and become plateau-like as $D a$ increases. However, the transition from pulse-like to plateau-like is a lot quicker than it is for smaller Le. Along the unstable branch, the solution remains pulse-like for large $D a$. 


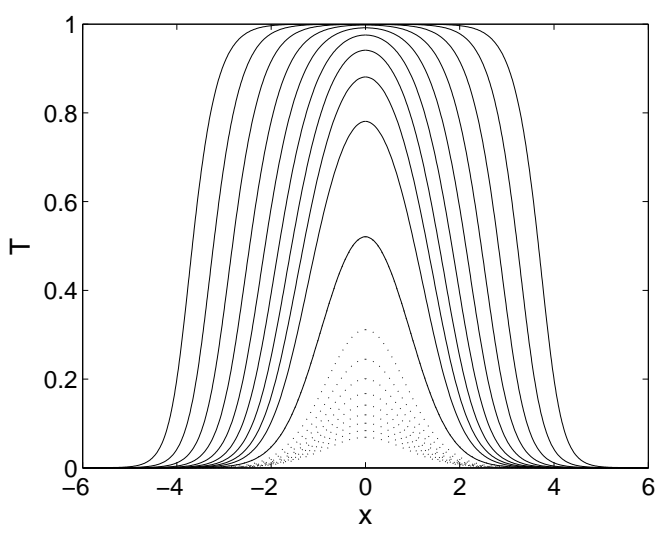

$(a)$

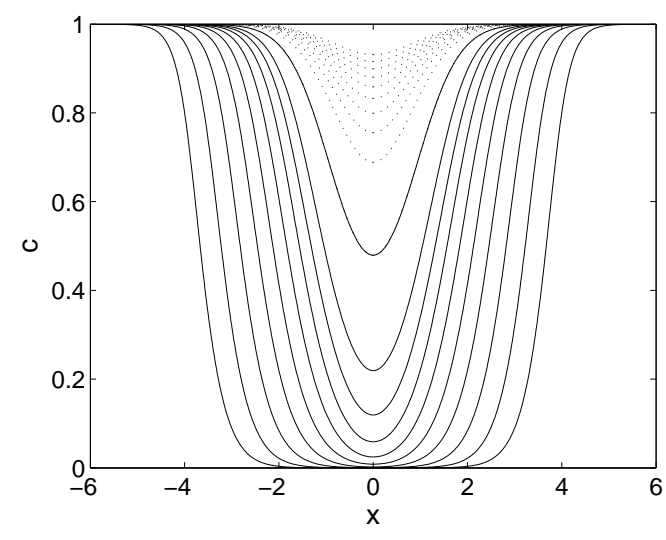

$(b)$

Figure 5: The steady solutions of the flame filament system (6)-(7) for $L e=1$. Both the stable solutions (denoted by solid lines) and the unstable solutions (denoted by dotted lines) are shown for logarithmically spaced values of $D a$ between $D a=6.963\left(\approx D a_{c}\right)$ and $D a=50$. (a) Profiles of T. (b) Profiles of $c$.

To model the behaviour of the solution near $D a_{c}$, we once again use bell-shaped test functions (9) as our basis functions. As seen in Fig. 6, near the saddle node we again find good agreement between the amplitude, $f_{0}$, and the inverse pulse width, $w$, obtained from the solution of equations (15)-(16) with these test functions, and the amplitude and inverse pulse width of $T$ obtained by integrating the full system (6)-(7). Note that for $L e=1$ we have $g_{0}=f_{0}$ and $v=w$ since $c=T$. However, the fit quickly deviates from the solution for the stable branch as anticipated. As with the case for small Lewis numbers, we see that the fit along the unstable branch remains very good even as we move further away from the saddle node. 


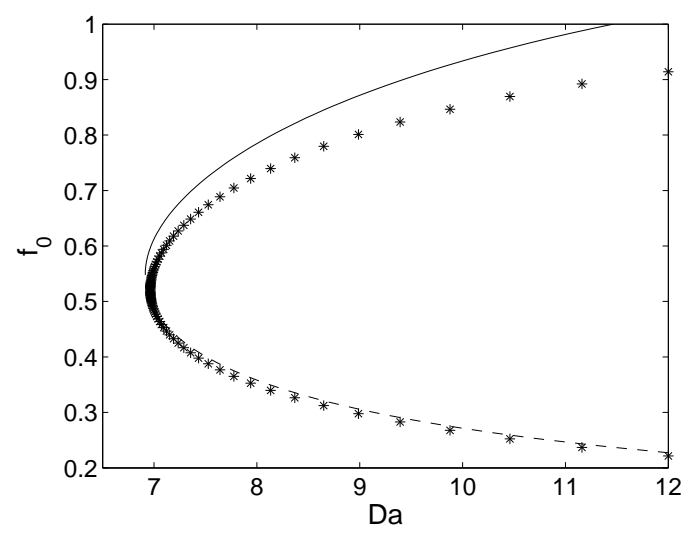

(a)

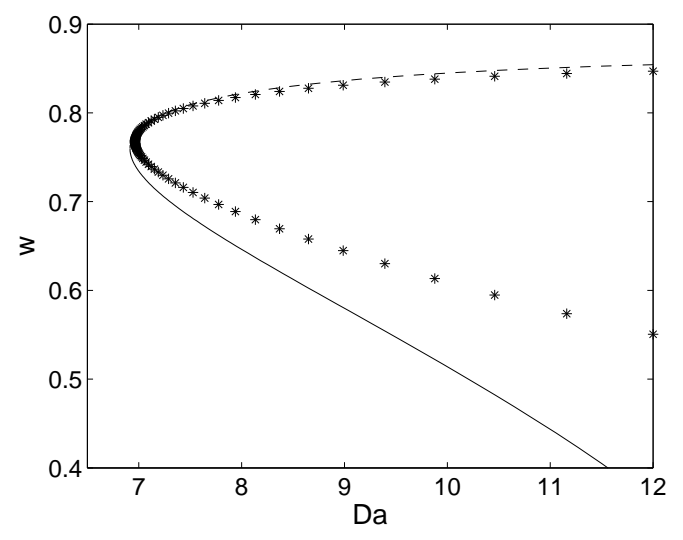

(b)

Figure 6: (a) Comparison of the numerical results for the amplitude of the temperature, $f_{0}$, obtained using the nonperturbative method with Gauss test functions (denoted by a solid line and a dashed line for the stable and unstable branches respectively), with the results for the full system of ordinary differential equations (6) and (7) obtained via shooting for $L e=1$ (denoted by stars). (b) Comparison of the numerical results for the inverse pulse width of the temperature, $w$, obtained using the nonperturbative method with Gauss test functions (denoted by a solid line and a dashed line for the stable and unstable branches respectively), with the results obtained via shooting for $L e=1$ (denoted by stars).

\subsection{Large Lewis numbers}

The large Lewis number regime is often called the "solid" regime. The heat conduction is much larger than the diffusivity of the reactant. This implies a broader reaction zone when compared to those corresponding to smaller Lewis numbers. The temperature within the product zone is less than the burnt temperature (i.e. in our rescaled model less than 1) and the reactant is consumed more than in the case of small Lewis numbers. Again, when increasing the Damköhler number, the temperature within the product zone will approach 1. In Fig. 7 we show the profiles of $T$ and $c$ for Le=10, obtained from the numerical solution of (6)-(7). We note that the profiles of both species broaden further close to the bifurcation point. On increasing $D a$ from $D a_{c}$, the profiles of the solutions very quickly change from pulse-like to plateau-like, and therefore the range of Damköhler numbers for which a bell-shaped function is a good approximation is diminished. We shall therefore consider the basis functions (9) as well as (10) to model the behaviour of the solutions near the saddle node. 


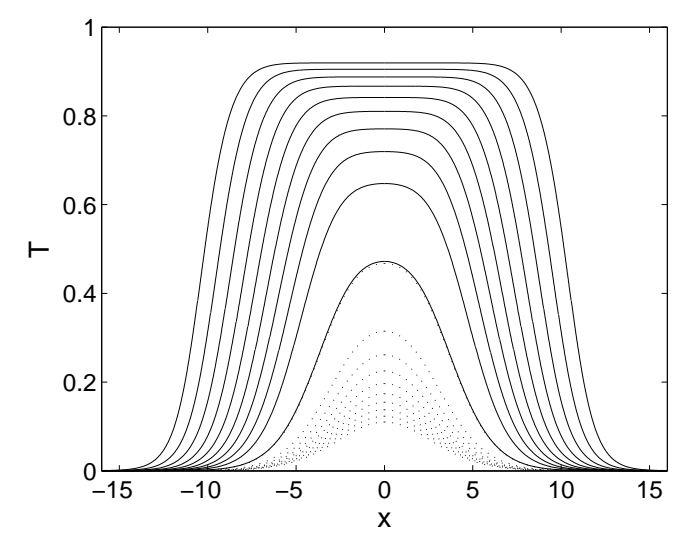

$(a)$

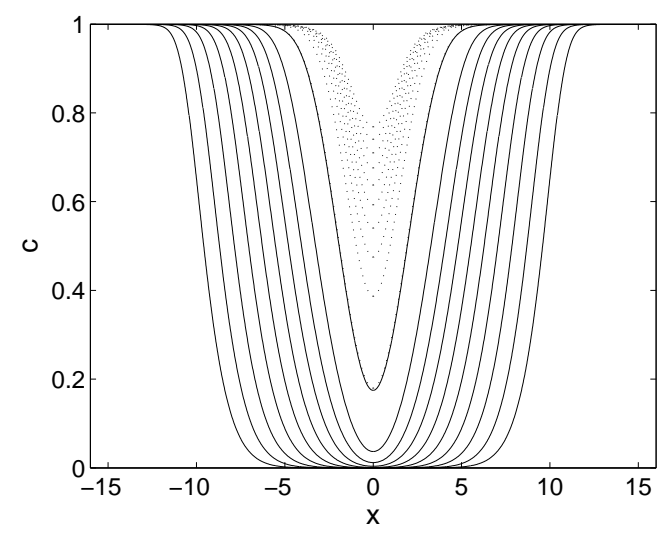

$(b)$

Figure 7: The steady solutions of the flame filament system (6)-(7) for $L e=10$. Both the stable solutions (denoted by solid lines) and the unstable solutions (denoted by dotted lines) are shown for logarithmically spaced values of $D a$ between $D a=8.462\left(\approx D a_{c}\right)$ and $D a=30$. (a) Profiles of T. (b) Profiles of $c$.

In Figs. 8 and 9 we see that the amplitudes and inverse pulse widths obtained using the nonperturbative method shows good agreement with the numerically obtained amplitudes near $D a_{c}$. Again the behaviour of the unstable branch is well approximated by using bell-shaped test functions. However, we see that in Fig. 8 the agreement of the amplitudes obtained by results from the test function approach (15)-(16) using bell-shaped test functions (9) with the respective amplitudes of $T$ and $c$ obtained by the simulation of the full system (6)-(7) is not as good for the stable branch as it is for smaller Lewis numbers. The same can be seen for the inverse pulse widths in Fig. 9. From Fig. 7 we see that the solution rapidly evolves into a plateau-like solution; we therefore use tanh-basis functions (10). In Fig. 8 it is seen that the amplitudes are much better recovered using the tanh-function ansatz (10). In Figs. 10 and 11 we show the results for the remaining four parameters needed to approximate the solution by tanh-functions as calculated by our nonperturbative test function method. To compare with solutions obtained by numerical simulations of the full system (6)-(7) we measured the distance from $x=0$ whereby the solution of $T$ and $c$ have fallen to three quarters and to one quarter of its maximal value at $x=0$. This yields two equations each for $T$ and $c$, which allows us to determine the parameters $w, \nu$ and $v, \mu$. Again the results are better for tanh-functions than the corresponding results for the Gaussian test functions depicted in Fig. 9. 


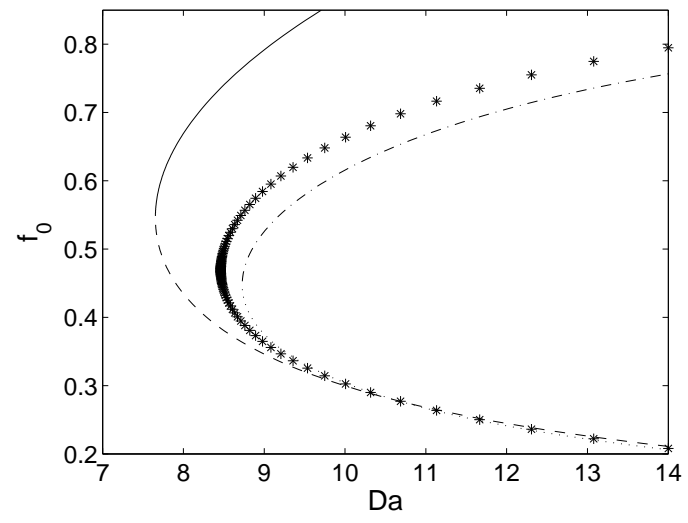

$(a)$

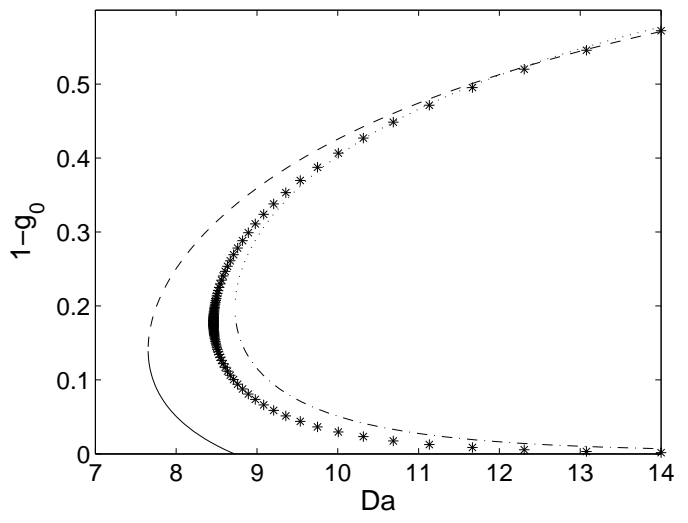

$(b)$

Figure 8: Comparison of the numerical results for the amplitude, obtained using the nonperturbative method with the basis functions for bell-shaped functions (9) (denoted by a solid line and a dashed line for the stable and unstable branches respectively) and the basis functions (10) (denoted by a dash-dotted line and a dotted line for the stable and unstable branches respectively), with the results for the full system of ordinary differential equations (6) and (7) obtained via shooting for $L e=10$ (denoted by stars). (a) The amplitude for the temperature, $f_{0}$, versus $D a$. (b) The amplitude for the concentration, $1-g_{0}$, versus $D a$.

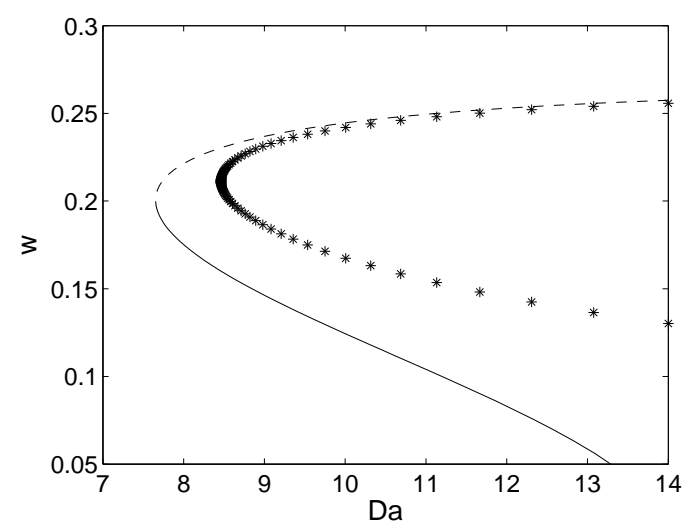

(a)

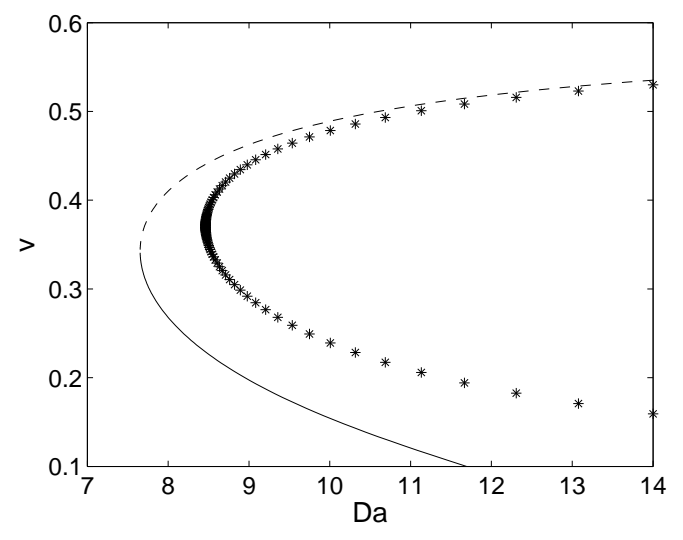

$(b)$

Figure 9: Comparison of the numerical results for the inverse pulse widths, obtained using the nonperturbative method with the basis functions for bell-shaped functions (9) (denoted by a solid line and a dashed line for the stable and unstable branches respectively), with the results for the full system of ordinary differential equations (6) and (7) obtained via shooting for $L e=10$ (denoted by stars). (a) The inverse pulse width for the temperature, $w$, versus $D a$. (b) The inverse pulse width for the concentration, $v$, versus Da. 


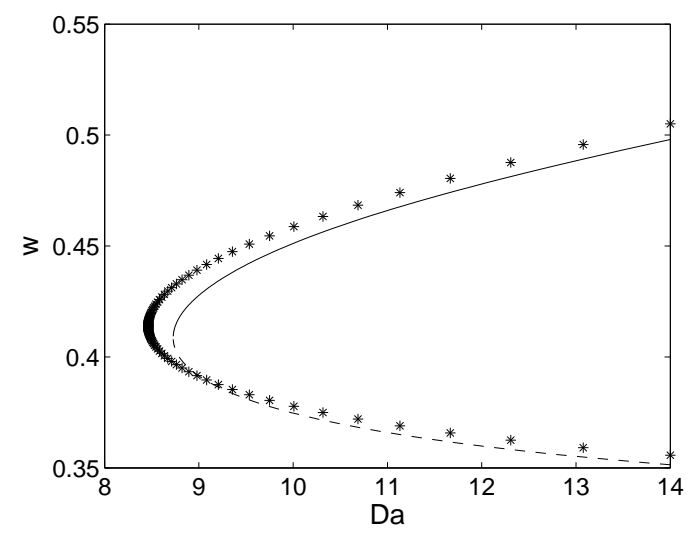

(a)

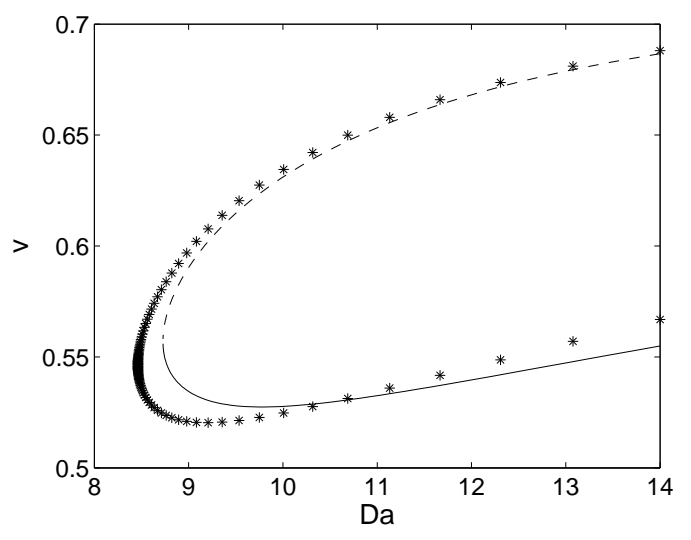

(b)

Figure 10: Comparison of the numerical results for the inverse pulse widths, obtained using the nonperturbative method with the basis functions for tanh-functions (10) (denoted by a solid line and a dashed line for the stable and unstable branches respectively), with the results for the full system of ordinary differential equations (6) and (7) obtained via shooting for $L e=10$ (denoted by stars). (a) The inverse pulse width for the temperature, $w$, versus $D a$. (b) The inverse pulse width for the concentration, $v$, versus $D a$.

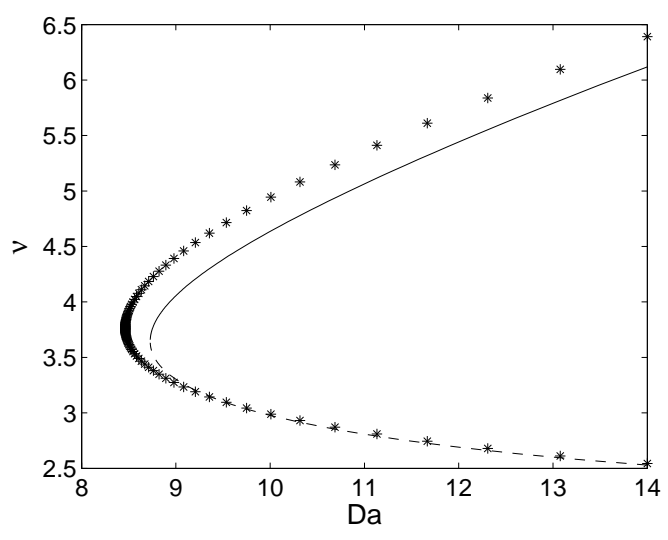

(a)

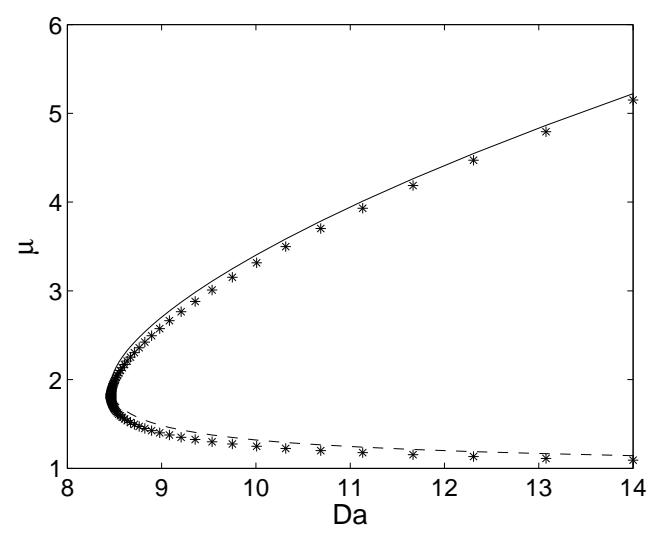

(b)

Figure 11: Comparison of the numerical results for the extent of the reaction zone, obtained using the nonperturbative method with the basis functions for tanh-functions (10) (denoted by a solid line and a dashed line for the stable and unstable branches respectively), with the results for the full system of ordinary differential equations (6) and (7) obtained via shooting for $L e=10$ (denoted by stars). (a) The extent of the reaction zone of the temperature, $\nu$, versus $D a$. (b) The extent of the reaction zone of the concentration, $\mu$, versus $D a$.

\subsection{The solution at the saddle node}

The solution of the flame filament system at the saddle node bifurcation depends on the value of the Lewis number. From a numerical analysis of the system (4)-(5), the value of 
$D a_{c}$ is seen to increase with $L e$. In Fig. 12, we show a comparison of the critical values of $D a$ calculated by the nonperturbative method with the aforementioned numerically obtained values of $D a_{c}$, over a range of Lewis numbers. We see that the critical Damköhler number does not vary much for large Lewis numbers. For small Lewis numbers we see that both the tanh-test functions (10) and the Gaussian test functions (9) approximate the critical Damköhler number very well. For larger values of $L e$, the tanh-test function ansatz is better suited to calculate $D a$, as we have already seen in Section 4.3, due to the rapid change of shape when varying $D a$.

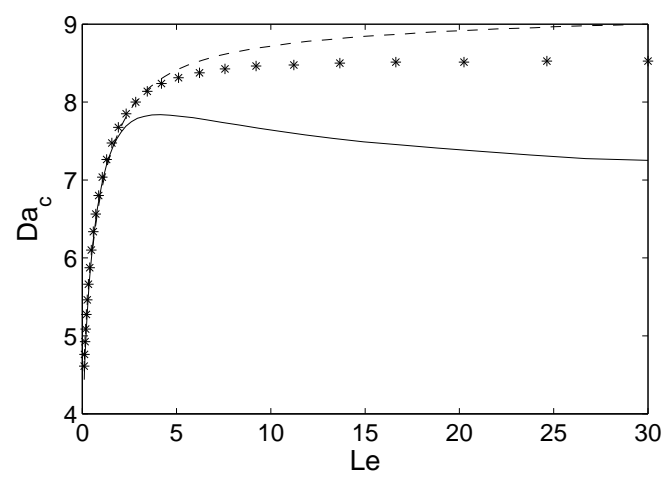

Figure 12: Comparison of the numerically obtained values of the critical Damköhler number (denoted by stars) obtained from integrating the full system (4)-(5), with the values of $D a_{c}$ obtained using the nonperturbative method with bell-shaped test functions (9) (denoted by a solid line) over a range of $L e$ and with a tanh test function (10) (denoted by a dashed line).

We note that the values of $D a_{c}$ calculated via the nonperturbative method approach a finite value for large $L e$. This is qualitatively similar to the behaviour of the numerically obtained values of $D a_{c}$ for large $L e$.

It has been shown in [18] that the critical Damköhler number depends very strongly on the parameter $\epsilon$ which is related to the inverse of the activation energy. In Fig. 13 we present a comparison of the functional dependency of $D a_{c}$ on $\epsilon$, which we obtain from the nonperturbative method using bell-shaped test functions (9), with the one obtained by numerically simulating the full system (6)-(7) at $L e=0.1$. From Sec. 4.1, we recall that such test functions were found to be a good approximation to the solution for $L e=0.1$ and $\epsilon=1$. As $\epsilon$ increases we see very good agreement between the two sets of values. 


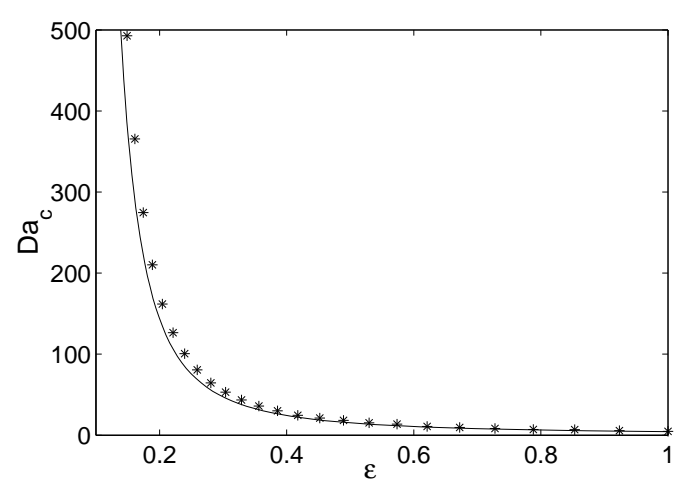

Figure 13: Comparison of the values of the critical Damköhler number, $D a_{c}$, at $L e=0.1$ obtained from integrating the full system (6)-(7) numerically (denoted by stars) with those obtained by the nonperturbative method using bell-shaped test functions (9) (denoted by a solid line), over a range of the parameter $\epsilon$.

\section{The solution far from the bifurcation point}

We recall that for $D a \gg D a_{c}$ the profiles of the stable solutions of $T$ and $c$ are plateau-like solutions with a narrow reaction zone. The profiles of the unstable solutions are pulse-like and, as we have shown in Sec. 4, they can be well described even for large Da using bell-shaped test functions (11). In this section, we are concerned with the stable branch.

It can be seen from Figs. 1, 5 and 7 that for large $D a$ the reactant is fully consumed in the central region of the filament, and the extent of the product zone in which the reactant is consumed increases with $D a$. This phenomenon is independent of the Lewis number. We also note that for large Lewis numbers and large Damköhler numbers the interior widths of the temperature and of the reactant zones product zone are equal and we have $\mu=\nu$. The amplitudes $f_{0}$ and $g_{0}$ both tend to 1 for large $D a$. This significantly simplifies the analysis of the solution. In the following we employ a phenomenological argument to determine the extent of the reaction zone $\mu$.

We now present an empirical formula for the wave speed $V_{0}$ of unstirred combustion fronts. For simplicity, we denote by $D a$ the reaction kinetics for the unstirred case as well. We measured the wave speed of the unstirred version of the system (4) using a shooting algorithm. We find that $V_{0}$ as a function of $D a$ clearly exhibits square-root behaviour. In particular we find that for large Lewis numbers $V_{0} \sim \sqrt{D a L e}$ is a good approximation. The scaling behaviour is reminiscent of an underlying autocatalytic structure for large $D a$; numerical simulations show that one can crudely approximate $c \approx 1-T$ and $K(T) \approx \alpha T$, where $\alpha$ is a (Lewis number dependent) constant. We determined $V_{0}(L e)$ numerically for several values of $D a(D a=100,500,1000,5000,10000)$. For large Le the quotient $V_{0} / \sqrt{D a L e}$ reaches a constant value of 0.669 with only very small deviation for the smaller values of the Damköhler number indicating that there is no Da-correction to the square-root behaviour of the velocity. However we can find a Lewis-number correction by 
employing linear regression. We find as an empirical formula for $V_{0}$,

$$
V_{0}=0.669 \sqrt{D a L e}\left(1-\frac{0.158}{L e}\right) \text {. }
$$

We have verified this equation by fitting to the velocity $V_{0}$ as a function of $D a$ for several values of a fixed Lewis number $(L e=1,10,25,50,1000,5000,10000)$. This formula is similar in construction to the formula for a different solution branch recently derived analytically in [31].

If we try to calculate the free parameters of the plateau-like test functions (10) from the algebraic equations (15)-(16) for the free parameters of the plateau-like test functions (10), we encounter difficulties. It turns out that the system of algebraic equations is degenerate. These problems seem to occur with systems where the plateau-solution connects a stable solution with an unstable one. In the Appendix we show analytical results for the tanhtest function method for a simple autocatalytic system which illustrates this degeneracy. However, for our system we may employ a simple phenomenological argument $[21,28]$ to calculate $\nu$ and $\mu$ for large Lewis numbers and large Damköhler numbers. Stationary fronts in lamellar systems arise from a balance between the diffusive front propagation prompted by the kinetic reaction term and the contracting $x$-dependent velocity due to the chaotic stirring. A balance of the front velocity $V_{0}$ and the velocity of chaotic stirring $x$ is reached when $V_{0}=x$, which implies that we get a stationary front when $V_{0}=\nu$. Hence we approximate

$$
\nu=0.669 \sqrt{D a L e}\left(1-\frac{0.158}{L e}\right) .
$$

In Fig. 14 we show that the phenomenological argument is indeed a good approximation for $\nu$ and $\mu$. 


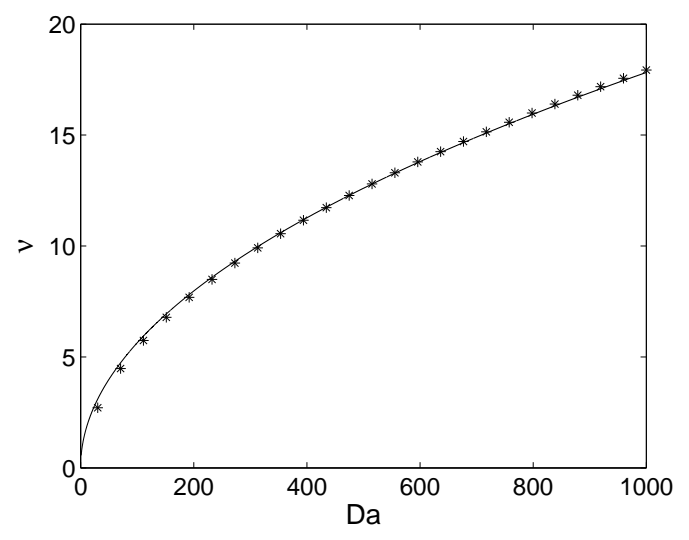

(a)

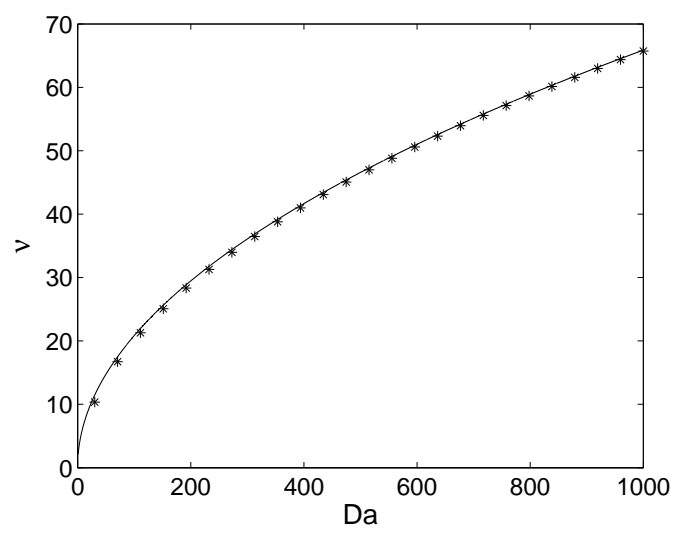

$(c)$

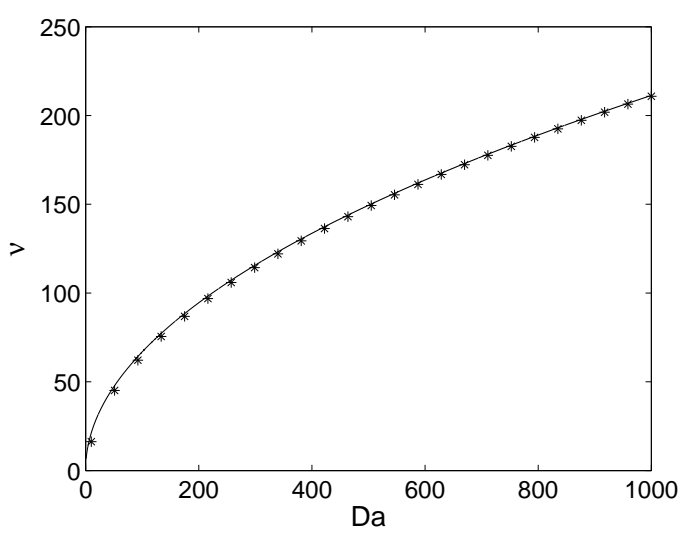

(e)

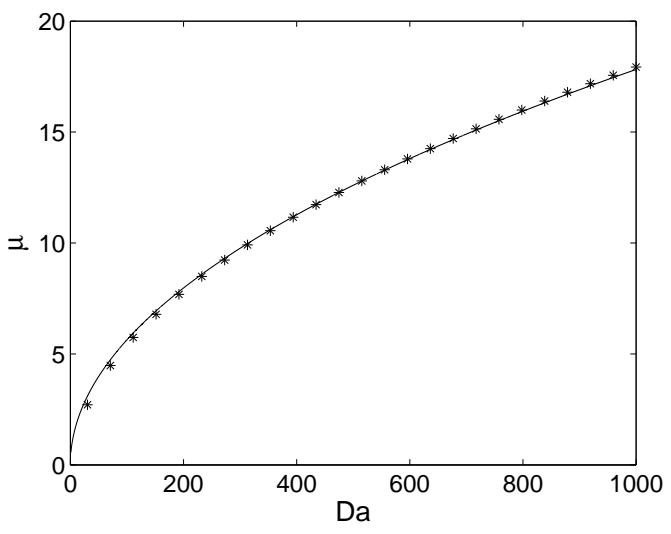

(b)

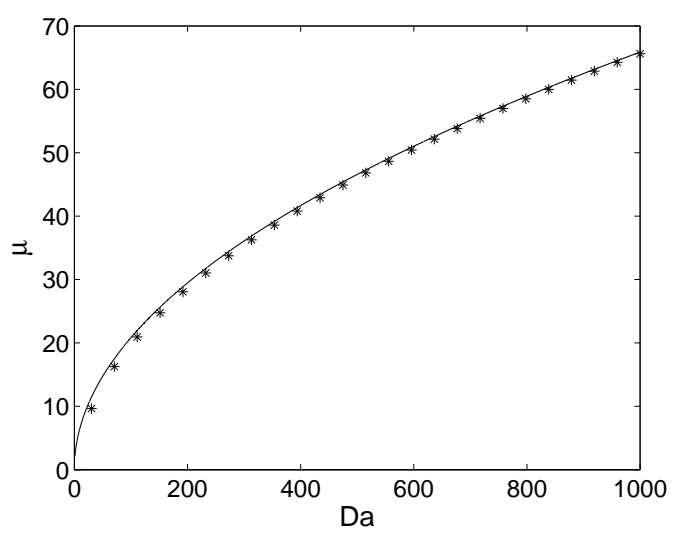

$(d)$

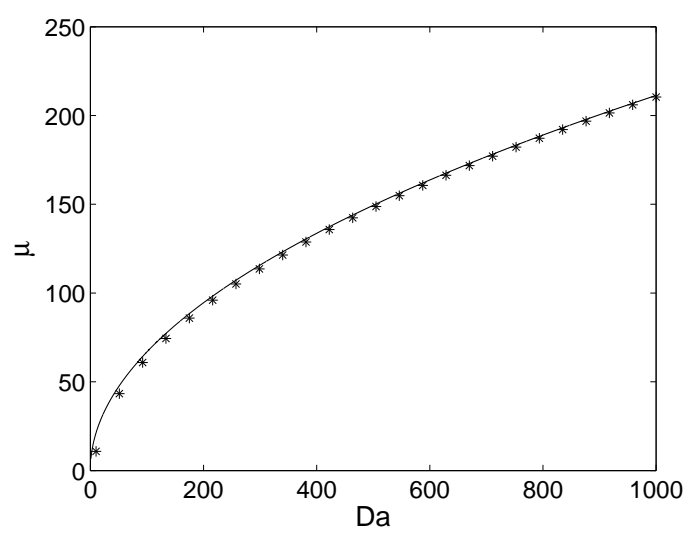

$(f)$

Figure 14: Comparison of the results for the extent of the product zone, obtained using the phenomenological formula (18) (denoted by a solid line), with the numerical results obtained from the solution of the system (4)-(5) (denoted by stars), for the width of $T$ (given by $\nu$ ), in the cases (a) $L e=1$, (c) $L e=10$ and (e) $L e=100$, and the width of $c$ (given by $\mu$ ), in the cases (b) $L e=1$, (d) $L e=10$ and (f) $L e=100$. 


\section{Summary}

We studied the effect of stirring on a combustion system. We used a reduced onedimensional filament model introduced in $[18,19]$ to describe the dynamics of the crossfilament structure. The effect of stirring is to remove heat from the filaments by quenching it. Subsequently the front does not contain enough heat to ignite the preheat zone. For small values of the Damöhler number, i.e. for large stirring rates, the reaction cannot be sustained. Only above a critical Damköhler number can nontrivial states form through the filaments. We used a nonperturbative method developed in [27], to determine the critical Damköhler number and also the actual solutions close to the Damköhler number. The critical Damköhler number $D a_{c}$ is not very sensitive to variations in the Lewis number provided it is large enough. This phenomenon is captured by our method. We found that the behaviour close to criticality can be well described using bell-shaped functions such as Gaussians (9) for small Lewis numbers, and for large Lewis numbers in the "solid" regime by tanh-test functions. The unstable solution branch can be well described using bell-shaped functions for all Lewis numbers. In Fig. 15 we show the actual solutions of the full problem (4) and (5) for $L e=1$ where we have $T=c$. We note that the case $L e=1$ is special in the sense that it allows us to use the test function method which otherwise fails far away from the bifurcation for tanh-functions due to a degeneracy (see Appendix). For $L e=1$, we can actually use the test function approach to calculate the inverse pulse width $w=v$ provided we also employ the phenomenological formula for the extent of the reaction zone $\nu=\mu(18)$, and also fix $f_{0}=1$.

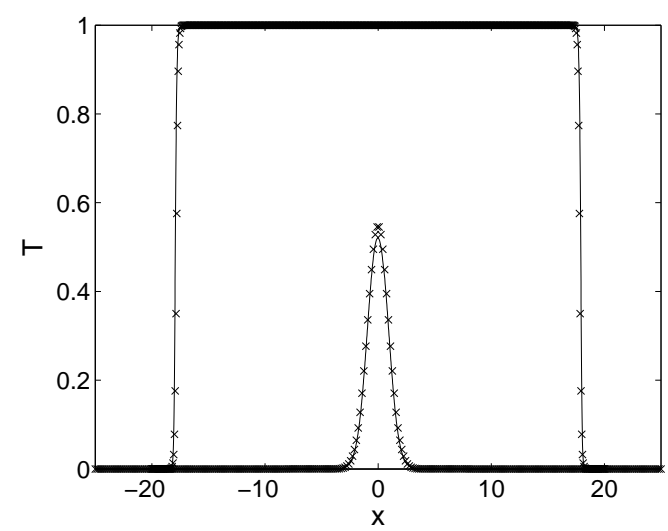

Figure 15: Comparison between numerical simulations of the PDE (4)-(5) and the test function method for $L e=1$. The plot shows a pulse like solution at $D a \approx D a_{c}$ and a plateau-like solution at $D a=1000$. Superimposed with crosses are the results of the test function method. We used Gaussian test functions (9) for the pulse like solution at the respective $D a_{c}$, and tanh-functions (10) for the plateau like solution. For the tanh-function ansatz $\nu$ is calculated by the phenomenological formula (18).

We then described the solutions for large Damköhler numbers. For small Lewis numbers the temperature approaches the burnt temperature $T=1$ from above, and higher 
temperatures can be reached in the product zone. For larger Lewis numbers the burnt temperature is approached for higher Damköhler numbers from above. We note that the largest change of the temperature with respect to the Damköhler number occurs close to the critical Damköhler number. For all Le, the concentration of the reactant $c$ reaches 0 within the product zone already slightly above the critical Damköhler number, corresponding to a full consumption of the fuel within the product zone. The extent of the product zone as a function of the Damköhler number was also described and a clear square-root behaviour was observed. A simple phenomenological argument allowed us to explain this dependence. We have determined an empirical formula for the wave speed of the unstirred combustion system for high Damköhler and high Lewis numbers. This empirical formula allowed us to determine the actual form of combustion fronts far away from the bifurcation with good accuracy for large Lewis numbers.

We notice that $V_{0}$ decreases (for a fixed Damköhler number) on decreasing the Lewis number. This can be seen from Fig. 14, where $\nu=V_{0}$ is shown. Now, in dimensional form, $L e=\kappa /\left(\rho C_{p} D\right)$, where $\rho, \kappa, C_{p}$ and $D$ are the respectively the density, thermal conductivity, specific heat capacity and molecular diffusivity of the fuel [30, 32]. Decreasing the Lewis number is equivalent to increasing the relative importance of $D, \rho$ and $C_{p}$ in relation to $\kappa$. Reducing $\kappa$ obviously decreases the ability of heat to propagate, and hence the combustion speed. Higher densities result in increased fuel mass in each location, which means more heat is needed in a given area to ignite all of the fuel before the wave moves on. Fuels with increased $C_{p}$ require more heat to increase the temperature by the a specified amount. Finally, increasing $D$ increases the transport of burnt fuel into the unburnt region and vice-versa, interfering with front propagation.

\section{A Autocatalytic System for large Damköhler num- bers}

Here we perform explicitly the calculations for the test function ansatz using tanh-test functions to describe the solution for large Damköhler numbers for the simple lamellar model of the autocatalytic Kolomogorov-Petrovsky-Piscounoff equation [33]

$$
\frac{\partial u}{\partial t}=D \frac{\partial^{2} u}{\partial x^{2}}+x \frac{\partial u}{\partial x}+D a u(1-u) .
$$

Again the solution is plateau-like for large Damköhler numbers, which motivates a tanhfunction as a test function. We write $u(x, t)$ in the form

$$
u(x, t)=\frac{1}{2} f \phi(\eta) \text { with } \eta=w x,
$$

where

$$
\phi(\eta)=\tanh (\eta+w \nu)-\tanh (\eta-w \nu) .
$$

We note that

$$
u_{x x}=\frac{1}{2} f w^{2} \phi^{\prime \prime}(\eta) \quad \text { and } \quad x u_{x}=\frac{1}{2} f \eta \phi^{\prime}(\eta) .
$$


We study steady-solutions of (19) using the test function (20) and consider the projections

$$
\begin{aligned}
\left\langle D u_{x x}+x u_{x}+D a\left(u-u^{2}\right) \mid u_{f}\right\rangle & =0, \\
\left\langle D u_{x x}+x u_{x}+D a\left(u-u^{2}\right) \mid u_{w}\right\rangle & =0, \\
\left\langle D u_{x x}+x u_{x}+D a\left(u-u^{2}\right) \mid u_{\nu}\right\rangle & =0 .
\end{aligned}
$$

Introducing $a=w \nu$ and $\psi(\eta)=\operatorname{sech}^{2}(\eta+a)+\operatorname{sech}^{2}(\eta-a)$ we can rewrite the projections as

$$
\begin{aligned}
-D w^{2}\left\langle\phi^{\prime 2}\right\rangle-\frac{1}{2}\left\langle\phi^{2}\right\rangle+D a\left(\left\langle\phi^{2}\right\rangle-\frac{f}{2}\left\langle\phi^{3}\right\rangle\right) & =0 \\
-\frac{w^{2} D}{2}\left(1+a \frac{\partial}{\partial a}\right)\left\langle\phi^{\prime 2}\right\rangle+\left(\left\langle\eta^{2} \phi^{\prime 2}\right\rangle+\nu w\left\langle\eta \phi^{\prime} \psi\right\rangle\right) & \\
+D a\left(a \frac{\partial}{\partial a}-1\right)\left(\frac{1}{2}\left\langle\phi^{2}\right\rangle-\left(\frac{f}{6}\right)\left\langle\phi^{3}\right\rangle\right) & =0 \\
-D \frac{w^{2}}{2} \frac{\partial}{\partial a}\left\langle\phi^{\prime 2}\right\rangle+\left\langle\eta \phi^{\prime} \psi\right\rangle+D a \frac{\partial}{\partial a}\left(\frac{1}{2}\left\langle\phi^{2}\right\rangle-\left(\frac{f}{6}\right)\left\langle\phi^{3}\right\rangle\right) & =0 .
\end{aligned}
$$

For large Damköhler numbers we note the scaling $\nu \sim \sqrt{D a}, w \sim \sqrt{D a}$ and subsequently $a \sim D a$. In the limit $D a \rightarrow \infty$, the inner products simplify significantly (see [29] for the explicit analytical expressions of the occurring inner products and their large $D a$-limits). At the leading orders in $D a$ we obtain

$$
\begin{aligned}
1-f & =0 \\
-\frac{w^{2} D}{3}+\frac{D a}{2}(1-f) & =0 \\
-\frac{2}{3} a+D a\left(1-\frac{2}{3} f\right) & =0 .
\end{aligned}
$$

This has the solution $f=1, w=0$ and $a=D a / 2$. For $D a \rightarrow \infty$ we therefore obtain

$$
\nu=\frac{a}{w} \rightarrow \infty
$$

Hence the solution tends to the stable state $u=1$, and not the observed plateau-like solution.

If we ignore the second projection (22) we can obtain the correct solution by employing the phenomenological argument that $\nu=V_{0}$ where $V_{0}=2 \sqrt{D a}$ [34]. The third equation (23) then yields $w=\sqrt{D a} / 4$ which is a good approximation for the large $D a$-limit [28].

Acknowledgements G.A.G. gratefully acknowledges support by the Australian Research Council, DP0452147. S.N.M was supported by a University of Sydney Postgraduate award. 


\section{References}

[1] Focus Issue: Active Chaotic Flow, edited by Z. Toroczkai and T. Tél, special issue of Chaos 12 (2002).

[2] I. R. Epstein, Nature 374, 321 (1995).

[3] T. Tél, A. de Moura, C. Grebogi and G. Károlyi, Physics Reports 413 91(2005).

[4] S. Edouard, B. Legras, F. Lefévre and R. Eymard, Nature 384, 444 (1996).

[5] E. R. Abraham, Nature 391, 577 (1998).

[6] A. P. Martin, J. Plank. Res. 22, 597 (2000).

[7] C. Lopéz, Z. Neufeld, E. Hernández-García and P. H. Haynes, Phys. Chem. Earth 26, 313 (2001).

[8] P. McLeod, A. P. Martin and K. J. Richards, Ecological Modelling 158, 111 (2002).

[9] A. P. Martin, Prog. Oceanograph. 57, 125 (2003).

[10] E. Hernández-García and C. Lopéz, Ecological Complexity 1, 253 (2004).

[11] N. Peters, Turbulent Combustion (Cambridge University Press, Cambridge, UK, 2000).

[12] W. E. Ranz, AIChE J. 25, 41 (1979).

[13] M. Abel, A. Celani, D. Vergni and A. Vulpani, Phys. Rev. E 64, 046307 (2001).

[14] P. Constantin, A. Kiselev, A. Oberman and L. Rhyzhik, Arch. Rational Mech. 154, $53(2000)$.

[15] P. Constantin, A. Kiselev and L. Rhyzhik, Comm. Pure Appl. Math. 54, 1320 (2001).

[16] A. Kiselev and L. Rhyzhik, Ann. I.H. Poincaré 18, 309 (2001).

[17] V. S. Santoro, A. Liñán and A. Gomez, Proc. Comb. Inst. 28, 2039 (2000).

[18] I. Z. Kiss, J. H. Merkin, S. K. Scott, P. L. Simon, S.Kalliadasis and Z. Neufeld, Physica D 176, 67 (2003).

[19] I. Z. Kiss, J. H. Merkin and Z. Neufeld, Physica D 183, 175 (2003).

[20] Z. Neufeld, Phys. Rev. Lett. 87, 108301 (2001).

[21] Z. Neufeld, P. H. Haynes and T. Tél, Chaos 12, 426 (2002).

[22] Z. Neufeld, C. Lopéz, E. Hernández-García and O. Piro, Phys. Rev. E 66, 066208 (2002). 
[23] E. Hernández-García, C. Lopéz and Z. Neufeld, Physica A 327, 59 (2003).

[24] T. Tél, T. Nishikawa, A. E. Motter, C. Grebogi and Z. Toroczkai, Chaos 14, 72 (2004).

[25] S. M. Cox, Physica D 199, 369 (2004).

[26] X. Z. Tang and A. H. Boozer, Chaos 9 (1999), 183-194.

[27] G. A. Gottwald and L. Kramer, Chaos 14, 855 (2004).

[28] S. N. Menon and G. A. Gottwald, Phys. Rev. E 71, 066201 (2005).

[29] S. M. Cox and G. A. Gottwald, Physica D 216, 175 (2006).

[30] F. A. Williams, Combustion Theory (Benjamin-Cummings, Menlo Park, CA, 1985).

[31] S. Balasuriya, G. A. Gottwald, J. Hornibrook and S. Lafortune, to appear in SIAM J. Appl. Math.

[32] R. O. Weber, G. N. Mercer, H. S. Sidhu, and B. F. Gray, Proc. R. Soc. Lond. A , 4531105 (1997).

[33] A. Kolmogorov, I. Petrovsky and N. Piscounoff, Bull. Univ. tat Moscow Ser. Int. A 1, 1 (1937).

[34] J. D. Murray, Mathematical Biology, 2nd edition (Springer Verlag, New York, 1993). 